\title{
Barrier Factors Related to COVID-19 Vaccine Literacy in Developing Countries: A Traditional Literature Review
}

\author{
Rani Tiyas Budiyanti ${ }^{1}$, Roro Isyawati Permata Ganggi ${ }^{2}$, and Murni Murni ${ }^{3}$ \\ ${ }^{1}$ Faculty of Public Health, Universitas Diponegoro, Indonesia \\ ${ }^{2}$ Faculty of Humanities, Universitas Diponegoro, Indonesia \\ ${ }^{3}$ Healthcare Practitioner, Semarang, Indonesia
}

\begin{abstract}
Coronavirus Disease-2019 (COVID-19) vaccination has been Coronavirus Disease-2019 (COVID-19) vaccination has been implemented in many countries involved in developing countries. However, many factors affected the implementation. One of them was the COVID-19 vaccine literacy. This research aims to know the barrier factors related to COVID-19 vaccination literacy in developing countries. This research method is a traditional literature review from journal articles in ProQuest, Scopus, Science Direct, Google Scholar, and EBSCOhost database, published in 2013 until 2021. The steps taken in the formal review were searched for specific keywords relevant to barrier factors related to COVID-19 vaccine literacy in developing countries, conducting a review, analyzing and critical appraisal, and writing a review. Based on the research, the barrier factors related to COVID-19 vaccine literacy in developing countries were low educational degree, lack of information access, lack of digital literacy, lack of valid information, and cultural perspective. Vaccine literacy can affect the success of the COVID-19 vaccination program, especially to achieve herd immunity coverage. The government must be concerned about improving COVID-19 vaccination literacy among the communities with multi-sector collaboration.
\end{abstract}

\section{Introduction}

Coronavirus Disease-2019 (COVID-19) is an infectious disease caused by coronavirus (SARS-CoV 2). COVID-19 can be transmitted through droplets so that the transmission is high-speed. WHO has stated COVID-19 as a Pandemic. A pandemic is an epidemic of disease that spreads on a wide scale. COVID-19 is stated as a pandemic because it has spread almost all over the world. WHO reported until April 16, 2021 there were 138,688,383 confirmed cases and caused 2,978,935 deaths worldwide [1]. Several efforts were made to suppress the spread of COVID-19, such as campaigns to implement health protocols. Health protocols that need to be done are wearing masks, avoiding crowds, and reducing mobility. Health protocol is a preventive effort but has not been able

\footnotetext{
* Corresponding author: ranitiyas@lecturer.undip.ac.id
} 
to suppress the adverse effect when a person is infected with COVID-19, so a vaccine is needed so that the effects of COVID-19 do not become more serious for sufferers [2].

A vaccine is a virus or bacteria that is still alive but has been disabled. COVID-19 vaccine is expected to stimulate the body to form immunity to SARS-CoV-2, so that if infected then a person will avoid severe pain and reach herd immunity [3]. The COVID-19 vaccination campaign faces several challenges including production, affordability, allocation, and deployment [4]. Some countries have carried out the campaign of COVID19 vaccine but in its implementation there is a rejection of vaccine, especially in developing countries in Asia. The rejection of vaccines especially in developing countries arises due to scepticism about the long-term effects and safety of vaccines [5]. Fake news trigger hesitance about vaccines conspiration also leads to this rejection [6]. Related to this, the authors try to dig further information related to factors that become a barrier in the literacy of COVID-19 vaccines in developing countries.

\section{Methods}

This research method is a traditional literature review. The selection of literature review methods aims to provide an overview of integrated and synthesized related knowledge of barrier factors related to COVID-19 vaccine literacy in developing countries. There are four steps taken in this literature review study, namely: (1) searching for specific keywords relevant to barrier factors related to COVID-19 vaccine literacy in developing countries from journal articles in ProQuest, Scopus, Science Direct, Google Scholar, and EBSCOhost database, which published in 2013 until 2021, (2) conducting a review, (3) doing analysis and critical appraisal, and (4) writing a review [7, 8].

\section{Results and discussion}

\subsection{COVID-19 vaccine literacy in developing countries}

COVID-19 vaccine literacy is the part of health literacy, which is knowledge, motivation, and competency to access, understand, and apply about COVID-19 vaccination and make a decision regarding COVID-19 vaccination to improve quality of life [9]. This literacy is a balance between individual, community and also population skills in complexity system. Literacy skills regarding COVID-19 vaccination are fundamental for people to process data and experience related to vaccination programs, improve critical and systemic thinking, and decide whether to accept the vaccination or not.

In China, COVID-19 vaccination has been implemented since December 2020 [10]. Based on research conducted by Wang et al. (2021) to 791 respondents longitudinally, information about vaccination and respondent literacy affects respondents' attitudes toward vaccination. This can be seen in a decrease in the desire for vaccination where in March 2020 it was $58.3 \%$, while in November and December 2020, it fell to 23.0\%. Most of the respondents said they wanted to postpone vaccination until the vaccine's safety could be ascertained. In addition, vaccination history, doctor's recommendations, convenience, and vaccine prices are also taken into consideration [10].

This condition also happens in Indonesia. Most of the respondents (65\%) agree with the COVID-19 vaccination, $27 \%$ of respondents are still unsure, and $8 \%$ rejected. Most people refuse vaccines because they still doubt their safety $(30 \%)$ and do not believe vaccination will be effective $(22 \%)$. Meanwhile, a minority of others stated that they did not believe in 
vaccines $(13 \%)$, feared vaccine side effects $(12 \%)$, religious reasons $(8 \%)$, and other reasons $(15 \%)$ [11].

Vaccines hesitancy also happen based on research in India to some medical students. COVID-19 vaccine was launched in India on January 16th, 2021, giving priority to health workers, including medical students. Based on 1068 medical students, 10,6\% still doubt with COVID-19 vaccine. The concerns about the safety and efficacy of a vaccine, rushed testing of vaccines before launched and lack of trust in government agencies [12].

\subsection{Barrier factors that related with covid-19 vaccine literacy}

COVID-19 vaccine information primarily related to safety and effectiveness are the most concerning things in vaccine acceptance. Information and research on this subject are continuously updated. However, there are many challenges related to COVID-19 vaccination literacy, such as lack of valid information and digital literacy, lack of information access, low educational degree, and cultural perspective.

\subsubsection{Lack of valid information and digital literacy}

Based on previous studies, most people who decide whether to reject or accept vaccinations are influenced by information about the level of effectiveness and safety of vaccines [13], so that valid information about vaccinations from reliable sources is essential in determining someone's decision. Doctor's recommendations, accessing official sources and massive health promotion regarding the safety of vaccinations are ways of overcoming vaccine safety hesitancy. Even so, digital literacy problems in communities, especially in developing areas, are still crucial. Much information, which is often referred to as an infodemic [14], makes it difficult for a person to sort out valid data and information and determine which data to trust, especially in the current era of the internet of things where data can be exchanged quickly either through the web, social media, or instant messaging. This is further exacerbated by the spread of hoax news related to vaccinations, such as conspiracy theories and so on [15].

The use of social media also increases the spread of misinformation related to vaccines. Some social media such as Instagram have tried to overcome this by providing valid link recommendations if someone posts about COVID-19 or COVID-19 vaccine. However, not all social media have done the same action [16].

Based on the research, more people in Indonesia, approximately $80 \%$ using social media to get information related to COVID-19, although this media is not so credible. On the other hand, fewer of them get information from a health professional that will preserve credible information about COVID-19 [11].

There is a Geneva-Switzerland based not-for-profit organization, namely Health on Net (HON), that validates reliable health information channels by providing the Health on Net Foundation Code of Conduct (HON Code) [17]. The principle of HON Code is authority, complementarity, confidentiality, attribution, justifiability, transparency of authorship, transparency of sponsorship, and honesty in advertising and editorial policy [18]. Although in 1995, 60 participants at an international health conference agreed to form a permanent body to promote the dissemination of accurate health information technology, this method has not been implemented yet in many countries, especially in developing countries. So that, people must carefully sort out valid and reliable sources of health data [19].

Therefore, the role of the state in providing valid and comprehensive health information is indispensable. In Indonesia, the right of the public to obtain valid and reliable health information is protected in Article 7 and Article 8 of Law Number 36 the year 2009 on Health [20]. Even so, there are still many obstacles and challenges in its implementation. 


\subsubsection{Lack of information access}

Besides the difficulty of accessing valid information, many developing countries have difficulties obtaining updated health information because of their limited access. Infrastructure development to support information technology has not been evenly distributed, causing society to rely on local health personnel and cadres to get health information. Although health personnel's level of information credibility is more valid and reliable, it will be a problem if the health resources are limited and distributed unevenly.

In this case, a strategy for disseminating COVID-19 vaccination information needs to be developed, such as using printed media in the form of brochures or leaflets that can be distributed to communities. The use of mass media to disseminate credible information related to vaccines also needs to be done. Involving community leaders also needs to be done so that health information can be conveyed in local community meetings [21].

\subsubsection{Low educational degree}

Valid and reliable information about the COVID-19 vaccine does not just make someone decide to receive the vaccine. After receiving information, someone will process the data and information through a thinking process. In this process, many possibilities can happen, such as information deletion, denials, generalizations, and logical fallacies. This condition will influence someone to imagine and make conclusions, beliefs, and determine actions.

Ability in the thinking process through systems and critical thinking needs to be developed. This is often related to a person's education level, although higher education does not always guarantee thinking critically and systematically [22]. Based on the research about knowledge, attitude, practice and perception of COVID-19 in many levels of education and profession in Bangladesh, a different perception was formed [23].

One of the challenges faced by developing countries is the low level of education and the habit of critical thinking [24]. In this case, providing valid health information is not enough. However, it is necessary to provide assistance in the thinking process and assistance to think about existing health issues.

\subsubsection{Cultural perspective}

In addition to information access, COVID-19 vaccination literacy is also influenced by local cultural perspectives and religious beliefs. Some of the resistance to vaccination arises from beliefs developed and passed down from generation to generation in society, such as the belief that the body is not allowed to accept chemical substances other than natural substances from nature [25]. This condition also can happen cause of a lack of trust in government and the creation of a mental model.

In this case, communication strategy and advocation must involve the community leader, a role model or respected people [26]. In addition, providing information about COVID-19 vaccination also can be inserted in ceremonies or cultural activities in society. It is necessary to introduce the vaccination history successfully in dealing with infectious diseases such as diphtheria, pertussis, and polio. The role of public health practitioners and health professionals are needed in this case to open the gap-health information barrier between community and health workers [27]. 


\section{Conclusion}

Based on the research, there are many barrier factors related to COVID-19 vaccine literacy, such as low educational degree, lack of information access, lack of digital literacy, lack of valid information, and cultural perspective. So in order to succeed in the COVID-19 vaccine campaign, there need to be efforts to improve the ability of individuals to detect fake news and health information, especially vaccine information, improve information access, improve critically and systems thinking habits, and involve community leaders in COVID-19 vaccine campaign.

\section{References}

1. World Health Organization. WHO coronavirus (covid-19) dashboard with vaccination data (2021). Retrivied from: https://www.who.int/emergencies/diseases/novelcoronavirus-2019/covid-19-vaccines/explainers

2. F. Haghpanah, G. Lin, S. A. Levin, E. Klein. Analysis of the potential impact of durability, timing, and transmission blocking of COVID-19 vaccine on morbidity and mortality. E. Clinical. Med. Lanc. 35, (2021) https://doi.org/10.1016/j.eclinm.2021.100863

3. P. Krause, T. R. Fleming, I. Longini et al. COVID-19 vaccine trials should seek worthwhile efficacy. Lancet. 396, 741-743 (2020). https://doi.org/10.1016/S0140$\underline{6736(20) 31821-3}$

4. O. J. Wouters, K. C. Shadlen, M. Salcher-Konrad, A. J. Pollard, H. J. Larson, Y. Teerawattananon, M. J. Challenges in ensuring global acess to COVID-19 vaccines: production, afforability, alocation, and deployment. Lanc. 397, 1023-1034 (2021). https://doi.org/10.1016/S0140-6736(21)00306-8

5. P. Malik, G. M. Leung. What can we expect from first-generation COVID-19 vaccines? Lancet. 396, 1467-1469 (2020). https://dx.doi.org/10.1016\%2FS0140$\underline{6736(20) 31976-0}$

6. V. Carrieri, L. Madio, F. Principe, F. Vaccine hesitancy and (fake) news: Quasiexperimental evidence from Italy. Health economics, 28 (11), 1377-1382 (2019). https://doi.org/10.1002/hec.3937

7. J. Jesson, L. Matheson, F. M. Lacey. Doing your literature review: Traditional and systematic techniques (2011)

8. C. Hart. Doing a literature review: releasing the research imagination (2018)

9. J. P. Michel. J. Goldberg. Education, healthy ageing and vaccine literacy. J. Nutr. Health. Aging. 25, 698-701 (2021). https://doi.org/10.1007/s12603-021-1627-1

10. J. Wang, X. Lu, X. Lai, Y. Lyu, H. Zhang, Y. Fenghuang, H. Fang. The changing acceptance of COVID-19 vaccination in different epidemic phases in China: A longitudinal study. Vaccines, 9 (3), 191 (2021).

https://doi.org/10.3390/vaccines9030191

11. Director General Disease Prevention and Control Decree Number HK 02.02/4/1/2021 about Technical Instructions for the Implementation of Vaccinations in Order Coping with Coronavirus Disease Pandemic 2019 (COVID-19)

12. J. Jain, S. Saurabh, A. D. Goel, M. K. Gupta, P. Bhardwaj, P. R. Raghav. COVID-19 vaccine hesitancy among undergraduate medical students: results from a nationwide survey in India. medRxiv (2021). https://doi.org/10.1101/2021.03.12.21253444 
13. K. Wang, E. L. Y. Wong, K. F. Ho et al. Change of willingness to accept COVID-19 vaccine and reasons of vaccine hesitancy of working people at different waves of local epidemic in Hong Kong, China: Repeated cross-sectional surveys. Vaccines, (2021), 9 (1), 62. https://doi.org/10.3390/vaccines9010062

14. F. Dib, P. Mayaud, P. Chauvin, O. Launay. Online mis/disinformation and vaccine hesitancy in the era of COVID-19: Why we need an eHealth literacy revolution. Human vaccines \& immunotherapeutics, (2021). 1-3. https://doi.org/10.1080/21645515.2021.1874218

15. Y. Wang, M. McKee, A. Torbica, D. Stuckler. Systematic literature review on the spread of health-related misinformation on social media. Social Science \& Medicine, 240, 112552 (2019). https://doi.org/10.1016/j.socscimed.2019.112552

16. C. M. Pulido et al. A new application of social impact in social media for overcoming fake news in health. International journal of environmental research and public health, 17 (7), 2430 (2020). https://doi.org/10.3390/ijerph17072430

17. Health On the Net Code of Conduct. HONcode: principles - quality and trustworthy health information. Retrieved from www.hon.ch

18. J. M. Ball. Better Health Through Informatics: Managing Technology to Deliver Value, in O'Carroll, Patrick W.; Yasnoff, William A.; Ward, M. Elizabeth; Ripp, Laura H. Martin, Ernest L. (eds.). Public Health Informatics and Information Systems. Springer Science \& Business Media (2006)

19. Health On the Net Code of Conduct. HONcode: Certification for Websites with Web 2.0 elements. Retrieved from www.hon.ch

20. Number 36 Year 2009 Concerning Health. Retrieved from https://www.hukumonline.com/pusatdata/detail/lt4af3c27570c04/undangundangnomor-36-tahun-2009

21. P. Rzymski, L. Borkowski, M. Drąg et al. The strategies to support the COVID-19 vaccination with evidence-based communication and tackling misinformation. Vaccines, 9 (2), 109 (2021). https://doi.org/10.3390/vaccines9020109

22. C. Angeli, N. Valanides. Instructional effects on critical thinking: Performance on illdefined issues. Learning and Instruction, 19 (4), 322-334 (2009). https://doi.org/10.1016/j.learninstruc.2008.06.010

23. M. Z. Ferdous, M. S. Islam, M. T. Sikder I et al. Knowledge, attitude, and practice regarding COVID-19 outbreak in Bangladesh: An online-based cross-sectional study. PloS one, 15 (10) (2020). https://doi.org/10.1371/journal.pone.0239254

24. J. E. Richmond. Bringing Critical Thinking to the Education of Developing Country Professionals. International education journal, 8 (1), 1-29 (2007). Retrivied from https://www.researchgate.net/profile/JohnHoge/publication/237456202_Establishing the need for_crosscultural and global issues research/links/55108ab20cf20352196c0c6d/Establishingthe-need-for-cross-cultural-and-global-issues-research.pdf\#page $=7$

25. J. A. Reich. Of natural bodies and antibodies: Parents' vaccine refusal and the dichotomies of natural and artificial. Social Science \& Medicine, 157, 103-110 (2016). https://doi.org/10.1016/j.socscimed.2016.04.001

26. J. J. Van Bavel, K. Baicker, P. S. Boggio et al. Using social and behavioural science to support COVID-19 pandemic response. Nature human behaviour, 4 (5), 460471(2020). Retrivied from https://www.nature.com/articles/s41562-020-0884-z

27. E. A. Harrison, J. W. Wu. Vaccine confidence in the time of COVID-19. Eur. J. Epidemiol. 35, 325-330 (2020). https://doi.org/10.1007/s10654-020-00634-3 